\title{
El controvertido papel del requisito de la autoconciencia en la evaluación moral del daño de la muerte
}

\author{
The controversial role of the requirement \\ of self-awareness in the moral judgement \\ of the harm of death \\ OLGA CAMPOS SERENA \\ Universidad de Granada (España)
}

Recibido: 02-09-2011

Aprobado definitivamente: 17-11-2011

\section{RESUMEN}

¿Podemos afirmar que una muerte indolora supone un daño para el individuo que muere? En este artículo se analiza el criterio más utilizado para responder afirmativamente a la pregunta. Un criterio que insiste en la necesidad de tener un interés por seguir viviendo. En el artículo pregunto si la autoconciencia requerida para la presencia del citado interés puede ser realmente condición necesaria en un análisis adecuado del daño de la muerte.

\author{
PALABRAS CLAVE
}

MUERTE, ANIMALES NO HUMANOS, AUTOCONCIENCIA,ANÁLISIS PRIVACIONISTA

\section{ABSTRACT}

Can we say that a painless death harms the individual who dies? This paper examines the criterion more frequently used to respond affirmatively to this question. It's an approach that emphasizes the need to have an interest in staying alive. Here I ask if the self-awareness required 
for the presence of such interest may actually be a necessary condition in a proper analysis of the harm of death.

\author{
KEYWORDS \\ DEATH, NON HUMAN ANIMALS, SELF-AWARENESS, \\ DEPRIVATIONIST ANALYSIS
}

LA PRETENSIÓN DE ESTE ARTÍCULO es evaluar las propuestas que defienden que sólo los individuos con un interés manifiesto en seguir viviendo son dañados cuando mueren. Igual que el hecho de tener un interés en no sufrir es lo que hace que alguien pueda ser dañado en el plano del sufrimiento. El núcleo común de las teorías analizadas pasa por entender, como condición de posibilidad para que la muerte sea algo malo, la capacidad de concebirse a uno mismo como un ente diferenciado existiendo en el futuro.

Cuando el nivel de conciencia de los distintos individuos tiene un papel tan determinante en el análisis resulta ineludible la pregunta por nuestras responsabilidades morales hacia los animales cuando se trata de la muerte de estos. Por ello considero especialmente relevante ver cómo han desarrollado sus análisis al respecto tanto Tom Regan desde una teoría de los derechos, como Peter Singer desde un utilitarismo de la preferencia. Y si en un caso se establece el criterio de ser sujeto de la propia vida y en otro el criterio de la personeidad, es fácil comprobar que en ambos estará en juego la autoconciencia como aquello que permite establecer una diferencia cualitativa entre los individuos que han de estar protegidos frente a la muerte y aquellos otros para los que se entenderá que esta no puede dañarles. Parece necesario, para tomar decisiones donde varias vidas están en conflicto, poder justificar una jerarquía no especieísta del valor de las vidas. Debido a ello sería lógico estar de acuerdo en que el objetivo del presente artículo no es baladí.

Las propuestas animalistas suelen insistir en que postular un principio de igualdad que abarque también a algunos animales no humanos ha de ser considerada una opción razonable. La igual consideración de intereses iguales resulta ser una buena forma de interpretar la idea de igualdad. Ello supone reconocer en muchos animales aquello que posibilita la presencia de intereses, esto es, la capacidad de sentir. Suele insistirse en que todos los que cumplimos este criterio somos iguales, en el sentido de que nuestros intereses deben contar igualmente siempre que sean los mismos. Pero poco se ha indagado en la cuestión de si ello supone a su vez que todos somos iguales en las situaciones en que entra en juego la muerte. 
Es sabido que las situaciones en las que tiene lugar la muerte de los individuos de la comunidad moral generan un tipo de decisiones en las que habrá que contar con muchos elementos. ${ }^{1}$ Pero podría pensarse que, si bien la presencia de un interés en no sufrir es lo que determina la necesidad de proteger el bienestar básico de un individuo, la presencia de un interés por seguir viviendo es lo que debería determinar la consideración de la muerte como un daño. Si se admitiera que es así habría que ver entonces qué es lo que determinaría la existencia de tal interés. Pero además surgiría una segunda cuestión y es la de si todos los que merecen tal protección moral han de estar protegidos de la misma forma. Esto supone preguntar por la forma en que habría que hacerse cargo de los conflictos entre las vidas de distintos individuos. Porque aunque pudiera concluirse que muchos animales no humanos merecen una protección moral frente a la muerte, podríamos perseguir justificar que la vida humana es más valiosa. En este contexto, y sin cuestionar el principio de igualdad, lo que lo anterior supone es mostrar la posibilidad de jerarquizar la fuerza del interés en continuar viviendo de los distintos individuos.

Lo que en un primer momento considero relevante es profundizar en la idea de que la preferencia por seguir viviendo es condición de posibilidad para poder admitir que la muerte supone un daño para el individuo que muere. Lo inmediato es preguntar por las capacidades que hemos de asociar a la presencia de la apuntada preferencia. Admitiré que no se trata de que ciertas capacidades hagan que los individuos que las poseen sean más valiosos desde un punto de vista objetivo, esto es, más valiosos por ese motivo en sí mismo. ${ }^{2}$ La clave está más bien en que ello es lo que hace que la muerte suponga un daño para el individuo que muere. Los defensores de este criterio parecen estar afirmando una teoría del daño en la que valorar $\mathrm{X}$ en sí mismo es necesario para poder sufrir el daño de no-X. ${ }^{3}$ Si se habla en términos de derechos morales habrá que entender que tal capacidad de valoración en un individuo hace que deba activarse una protección moral para su vida.

Necesariamente habría que preguntar por las distintas interpretaciones posibles de la capacidad de valorar la vida. Parece haber un elemento común que tiene que ver con la habilidad para valorar posibilidades futuras. Lo que habrá

1 Cuando pregunto por el daño que supone la muerte dejo al margen posibles cuestiones relacionadas con el sufrimiento que puede acompañar al proceso de morir.

2 Si interesara conocer al respecto una propuesta más objetivista puede consultarse R. Frey 1987.

3 El argumento que relaciona esta argumentación con la atribución de derechos morales tendría la siguiente forma. Véase S. F. Sapontzis 1987, pp. 161-175. Sólo los seres capaces de valorar X en sí mismo pueden sufrir el daño de no-X. Sólo los seres capaces de sufrir no-X como un daño pueden tener un derecho moral a X. S es incapaz de valorar X en sí mismo. Entonces $\mathrm{S}$ no puede tener un derecho moral a $\mathrm{X}$. 
que determinar es cuán futuras o complejas han de ser tales posibilidades. Frente a una interpretación más exigente ${ }^{4}$ podría defenderse una concepción menos estricta de lo que supone la apuntada valoración. Para ello habría que poner el acento en la necesidad de una preferencia en continuar viviendo admitiendo que ello no implica una comprensión explícita de lo que supone morir. No obstante sí sería una condición necesaria la presencia de determinadas características psicológicas, por ejemplo, ser consciente de uno mismo como entidad distinta y concebirse existiendo a lo largo del tiempo. Estas sí resultan imprescindibles para poder tener la preferencia señalada..$^{5}$

Me propongo evaluar el éxito de esta propuesta que he descrito como menos exigente. Desde ella se insiste en que, para afirmar que la muerte daña al que muere, debe darse un cierto grado de autoconciencia, admitiendo que ello no implica una comprensión humana del hecho de la muerte. De ahí la pertinencia de prestar atención a los análisis que de esta cuestión han llevado a cabo los autores animalistas.

Antes de analizar la primera propuesta particular merece la pena situar el contexto normativo en el que esta se enmarca. Hemos de saber que la idea de dignidad ha jugado un papel determinante en la consideración de las obligaciones morales. Ya en la cultura clásica se hablaba de dignidad para denotar un cierto tipo de carácter. A partir de la Edad Media esto comienza a cambiar y ahora se trata de algo universal, inherente al ser humano. Pero desde el Renacimiento recobran importancia los rasgos de carácter que previamente se asociaban a la dignidad. La doctrina que Kant elabora en torno a los fines y los medios de los actos humanos es representativa. Dicha doctrina descansa en la idea de que fin es lo que sirve a la voluntad de fundamento objetivo, mientras que aquello cuyo efecto es el fin se llama medio (I. Kant 1988, pp. 81-84). Los fines equivalen a motivos objetivos que se autoimponen a todo ser racional y se oponen a aquello que proviene de los deseos y las inclinaciones. Si todo ser racional existe como fin en sí mismo, los seres irracionales tienen sólo un valor relativo y hay que considerarlos como medios. Debido a ello se mantiene que no tenemos ningún deber moral directo hacia los últimos.

4 Puede verse en este sentido la propuesta de R. Cigman, que reivindica la necesidad de contar con un tipo especial de deseo que la autora llama deseo categórico y que se asocia a un grado muy alto de reflexividad. Consúltese R. Cigman 1976, pp. 150-152 y 1981, pp. 48-62.

5 Resulta iluminador tener clara la posible distinción entre autoconciencia (self-consciousness) y self-awareness (autopercepción). Hay individuos conscientes de que existen a lo largo del tiempo (autopercepción) sin tener la capacidad de evaluar desde un punto de vista moral sus actos (autoconciencia). Véase P. Singer 1999a, p. 220. 
Analizaremos la propuesta de Tom Regan que, si bien asume el punto de vista de la teoría de los derechos, ofrece una interpretación distinta de los postulados normativos. Ello le permite justificar la inclusión de los animales en la esfera moral.

Regan habla del valor inherente para referirse a aquello que caracteriza a los titulares de derechos morales. El valor inherente no puede entenderse como algo que depende del esfuerzo de los individuos o del grado en que sean útiles. Una vez que se reconoce tal valor, admite, ya no estamos ante un asunto de grados. El autor habla del criterio de «ser sujeto de la propia vida» para referirse a algo semejante a la autoconciencia y que será lo que determine cuándo la muerte es un daño. Este criterio implica más que estar vivo y más que ser meramente consciente; supone tener creencias y deseos; percepción, memoria y sentido del futuro; una vida emocional junto con sensaciones de placer y dolor; preferencias e intereses de bienestar; habilidad para iniciar una acción en persecución de una meta; mantenimiento de una identidad psicológica; y también un bienestar individual, en el sentido de poder experimentar cómo es la vida de uno independientemente de su utilidad para otros (T. Regan 1983, pp. 243-248).

La asunción teórica del postulado del valor inherente es primaria. El criterio de ser sujeto de la propia vida se introduce con el objetivo de justificar la previa atribución de valor inherente. Frente a un enfoque fáctico, donde para decidir qué individuos poseen derechos se trata de establecer un conjunto de características no-morales (verificables empíricamente), el autor opta por un enfoque moral ( $\mathrm{T}$. Regan 1999, pp. 18-29). Desde aquí la decisión se toma sobre la base de juicios morales que reflejen la lógica del vínculo-moral. Este vínculo establece que determinado trato de algunos individuos es malo, cuando ello tiene como objeto meramente que se beneficien otros. El criterio sirve para señalar una similitud relevante entre agentes y pacientes morales. Así, el hecho de que compartan las características que determina el criterio permite justificar la atribución de valor inherente también a los pacientes morales. Es así porque el principio formal de justicia exige tratar casos similares de igual forma. ${ }^{6}$ Un mismo trato que debe extenderse hasta asegurar que, para todos los poseedores de valor inherente, se establece una adecuada protección moral frente a la muerte.

En este esquema normativo tal protección se traducirá en un derecho moral a la vida. Puede comprobarse entonces que, según estos postulados, habría

6 La interpretación del mismo viene dada para Regan por el principio del respeto, que se deriva del postulado del valor inherente. Este principio exige que tratemos a los individuos (agentes y pacientes) de forma que se respete su valor inherente. Se materializa en un derecho (el derecho a un tratamiento respetuoso) que incluye en un plano más específico el derecho a la vida. Consúltese T. Regan 1983, pp. 248-286. 
una diferencia de tipo cualitativo entre los que cuentan con dicho derecho y los que no.

Pero Regan es consciente de la necesidad de poder establecer una jerarquía que permita elegir la violación de derechos menos mala cuando haya conflictos entre vidas protegidas moralmente. Para ello toma como punto de partida la teoría de los deberes condicionales o «prima facie» (W. D. Ross 1930). La idea es que las decisiones éticas deben tomarse realizando un balance de los diferentes deberes implicados en cada situación concreta. Así, por ejemplo, afirmar que el derecho de ser tratados con respeto es un derecho de este tipo implica defender su relevancia moral. Ello supone admitir que quien quiera dañar a alguien debe justificar su acción apelando a otros principios morales válidos y mostrando que en ese caso tales principios pesan más moralmente que el derecho a no ser dañado.

Para Regan las situaciones en las que está en juego la prevención de daños serían un buen ejemplo del tipo de casos en los que podría darse la anterior justificación. Se trataría de determinar si es legítimo dañar a un individuo inocente cuando con ello evitamos el daño de un gran número de inocentes. Dicho de otra forma, habría que preguntar acerca de si los números cuentan o no. Hay que distinguir, afirma Regan, entre aquellos casos en los que se da un sufrimiento comparable y otras situaciones en las que se da un sufrimiento no comparable. La toma de decisiones ha de venir determinada por el hecho de si se da un daño de uno u otro tipo. Obviamente hemos de saber cuándo dos daños son comparables según el autor. Su respuesta es que lo son cuando quitan la misma cantidad de bienestar de un individuo, o de dos o más individuos. Para este tipo de casos Regan propone regirse por el principio llamado «the miniride principle», esto es, «Principio de la minimización de la desatención» (O. Horta 2007, p. 986). Sin embargo, cuando nos encontremos en una situación donde se dé un sufrimiento no comparable debemos guiarnos por el conocido «the worse-off principle» $\mathrm{O}$ «Principio del más perjudicado» (O. Horta 2007, p. 986). Ambos son derivables del principio del respeto, y tienen la forma siguiente ( $\mathrm{T}$. Regan 1983, pp. 286-327):

Principio de la Minimización de la Desatención: dejando a un lado consideraciones especiales, siempre que tengamos que elegir entre invalidar los derechos de una mayoría o los de una minoría de inocentes, y en el caso en el que cada uno de los individuos afectados se vea perjudicado en grado comparable, deberíamos escoger invalidar los de la minoría antes que los de la mayoría. Lo que implica este principio es que, en los casos de daños comparables, los números cuentan.

Principio del más Perjudicado: dejando a un lado consideraciones especiales, siempre que debamos decidir si hay que invalidar los derechos de una 
mayoría o los de una minoría de inocentes, y en el caso en el que el perjuicio afrontado por la minoría les haga estar peor que cualquiera de la mayoría lo estaría si escogiésemos cualquier otra opción, deberíamos, en consecuencia, invalidar los derechos de la mayoría. De forma que, en los casos de daños no comparables, los números no cuentan.

Partiendo de este análisis en torno a la prevención de daños lo que me interesa es ver cómo resolvería Regan una situación de conflicto entre el derecho a la vida de distintos individuos. Ya señalé que en la identificación de aquellos que tendrán un derecho moral a la vida el autor asume un enfoque cualitativo, en tanto que la posesión de valor inherente marca una diferencia no sólo cuantitativa con aquellos que carecen de tal tipo de valor.

De lo que se trata ahora es de ver cómo se podría establecer una jerarquía entre aquellos que cuentan con un derecho a la vida. Es sabido que Regan ha reivindicado una concepción no absoluta de los derechos. El siguiente paso, según él mismo establece en la argumentación, sería determinar si estamos antes daños comparables o no comparables. Teniendo en cuenta sus análisis en torno a la imposibilidad de establecer una gradación del valor inherente tendría que admitir que el derecho a la vida sería el mismo en todos los casos en que se posea dicho valor. Se estaría asumiendo que el daño al que daría lugar la muerte sería igual para todos ellos. Se trataría por tanto de daños comparables y la decisión vendría guiada por el Principio de la minimización de la desatención.

Quiero analizar el ejemplo que pone el propio Regan para ilustrar una situación de este tipo. Cuatro humanos normales y un perro se encuentran, tras un naufragio, en un bote salvavidas. El problema es que el bote se hundirá con más de cuatro individuos a bordo. La acción que el principio señalado prescribiría sería la de tirar a alguno de los ocupantes por la borda. Si la muerte supone el mismo grado de daño para todos los individuos con valor inherente y por tanto igual derecho a la vida (según su propuesta tendríamos que admitir que los cinco individuos del bote lo poseen), entonces habría que admitir que no tenemos razones para optar por tirar a alguno en concreto (obviando posibles razones indirectas). Se trataría de daños comparables según los postulados del autor, y el Principio de la minimización de la desatención dice que hay que prescindir de uno de los ocupantes para salvar a cuatro, pero no nos dice de cuál.

Sin embargo Regan insistirá en que su teoría es capaz de justificar la opción de tirar al perro. Afirma que su muerte provocaría un daño menor comparado con el daño que provocaría la muerte de cualquiera de los cuatro humanos. Esta solución puede coincidir con nuestras intuiciones prerreflexivas pero resulta incompatible con los presupuestos teóricos del autor. El derecho a la vida será igual de fuerte en el caso de todos sus poseedores porque ha insistido en que el valor inherente no admite grados, de forma que Regan no podría 
justificar que la muerte del perro provoca un daño menor. Su teoría no admite un análisis cuantitativo en este sentido. Resulta incoherente pretender afirmar ahora que la situación del bote salvavidas se sitúa en un contexto de daños no comparables.

Es fácil deducir las razones por las que Regan entiende que la muerte del perro supone un daño menor. Ello es algo que coincide con nuestras intuiciones. Resulta indudable la necesidad de un criterio cuantitativo que permita establecer una gradación entre los distintos individuos con derecho a la vida, algo que no parece compatible con los postulados teóricos de la teoría de Regan. Resulta problemática la pretensión de aplicar la idea de daños no comparables al contexto de la muerte si, como el propio Regan explica, por definición el postulado del valor inherente lleva a adscribir el mismo derecho a la vida. La reivindicación de un enfoque moral en la identificación de los individuos que tienen derechos hace que así sea. Todos los individuos con valor inherente, afirmaba, tendrán que estar protegidos igualmente frente a la muerte. Si esto es lo que debería deducirse del enfoque moral al que Regan apela al principio, la solución ofrecida para el caso del bote salvavidas nos lleva a pensar que en algún momento ha pasado a defender un enfoque fáctico donde lo que cuentan son determinadas capacidades.

Quiero insistir en que, de mantener el esquema teórico en el que se sitúa el autor, resultará muy problemática la pretensión de asumir un análisis cuantitativo del grado de daño que supondrá la muerte de los distintos individuos con derecho a la vida. ${ }^{7}$

III

El siguiente paso es valorar si es más exitosa al respecto la propuesta de otro importante autor animalista, Peter Singer. Este autor si sitúa en un contexto utilitarista desde el que quiere justificar la existencia de obligaciones morales más allá de los seres humanos. Es fácil ver las herramientas teóricas de la ética utilitarista para poder justificar una ampliación de la comunidad moral. Es una propuesta universalista porque tiene en cuenta los intereses de todos aquellos afectados por una acción, independientemente de cuestiones moralmente irrelevantes como el sexo, la raza e incluso la especie. Es bienestarista porque define lo que es éticamente bueno en términos del bienestar de los individuos. Es consecuencialista porque evalúa la corrección o incorrección de una acción en función de las consecuencias esperadas para dicha acción, esto es, en fun-

7 Además el Principio del más perjudicado puede llevar en sí mismo a resultados indeseables, y si no es así será porque el cálculo consecuencial ha entrado en acción haciendo que se pierda la identidad teórica de la teoría de los derechos. Véase D. Jamieson 1990, pp. 349-362. 
ción del grado en que ésta satisface intereses. Por último es agregativo porque suma los intereses de todos aquellos afectados por una acción (J.J. C. Smart y B. Williams 1973). El utilitarismo se fundamenta en la idea de que el dolor es algo a evitar y el bienestar algo a fomentar. De forma que, si los animales también tienen intereses, éstos deben contar en la evaluación de la misma forma que los del resto de individuos.

M. Nussbaum ha querido insistir en que las teorías de corte utilitarista no tienen la exclusividad en este sentido. La autora reivindica la viabilidad de interpretar la cuestión de los animales como una cuestión de justicia, proponiendo la «ética de las capacidades». Si el objetivo de la justica es garantizar que distintas clases de seres tengan oportunidades para actuar conforme a sus capacidades, entonces lo lógico sería asegurarse de que los principios que elaboremos incluyeran también a aquellos humanos (como los discapacitados cognitivos) y no humanos (como los animales) que no pueden participar en el establecimiento de los mismos (M. Nussbaum 2007, pp. 327-337, 352-400).

$\mathrm{Su}$ objetivo es establecer determinados derechos fundamentales, para que los actores políticos puedan encargarse de la justicia básica y no tengan que perseguir la maximización del bien total. Esto último, advierte, es uno de los motivos que deberían llevarnos a reconocer la validez de su propuesta alternativa, y es que esta no nos obliga a disponer de un concepto comprehensivo particular de bien.

Sin embargo en este sentido no puede pasarse por alto que Singer, más allá del utilitarismo clásico, defiende un utilitarismo de la preferencia. Lejos de una interpretación del bienestar como estados mentales placenteros reivindica una ampliación del concepto donde cuente también un elemento externo al sujeto, como la realización de sus preferencias o deseos. Singer nos propone adoptar la igual consideración de intereses. Hay que reconocer la relevancia del hecho de que existan seres con deseos y preferencias, e insiste en que nuestra capacidad para razonar nos puede mostrar algo que puede considerarse incuestionable: el sufrimiento de otro ser es muy similar al que yo mismo experimento y normalmente a ambos nos importa por igual evitarlo. Asumir esta perspectiva, admite, es el mayor grado de objetividad al que se puede llegar (P. Singer 2002, pp. 309-312). Ello posibilita un fundamento objetivo para la ética y proporciona un primer propósito independiente de nuestros propios deseos: la reducción del dolor y el sufrimiento. Si bien pueden reconocerse otros valores relevantes (la felicidad, por ejemplo), el de la reducción del dolor y el sufrimiento se presenta como el más urgente y menos discutible.

El autor reivindica una evaluación del valor de la vida en la que los individuos más conscientes se encuentren en una posición privilegiada. Lo que habrá que ver es si la diferencia estará en función de la presencia de una mayor 
cantidad de experiencias o la clave estará en el hecho de poder experimentar de manera cualitativamente diferente. ${ }^{8}$

Singer está interesado en fundamentar la idea de que matar a un individuo autoconsciente es peor que matar a un ser meramente consciente. Quiere insistir en que, desde marcos teóricos diferentes, habitualmente se ha mantenido que la muerte de las personas supone un daño desde el punto de vista moral (P. Singer 1995, pp. 112-126 y 1998, pp. 221-225). La adopción de un análisis cualitativo parece cobrar fuerza cuando Singer defiende que hay que entender de manera paralela la distinción entre individuos conscientes/autoconscientes y entre no personas/personas. ${ }^{9}$ El que alguien pueda ser considerado una persona dependerá de si puede alcanzar el grado de autoconsciente..$^{10} \mathrm{Y}$ lo que parece estar queriendo decir es que sólo la muerte de las personas puede ser considerada un daño. En un contexto utilitarista, reivindicar una concepción de lo bueno donde no sólo cuente los estados mentales placenteros sino también algo externo, como la satisfacción de preferencias, permitirá considerar como algo intrínsecamente malo el hecho de quitarle la vida a un individuo que posea una preferencia en continuar viviendo.

Dejando al margen razones indirectas, lo que parece desprenderse de su análisis es la idea de que la muerte de los individuos meramente conscientes es algo moralmente irrelevante. Si los autoconscientes son los únicos a los que la muerte daña entonces también serán los únicos con protección moral al respecto. Para los demás será legítima la aplicación del argumento de la reemplazabilidad.

Desde este argumento se defiende que no hay nada malo en prescindir de la vida de cualquier individuo consciente que haya tenido una existencia placentera, siempre que sea reemplazado por otro cuya vida contase al menos con la misma cantidad de satisfacción. Si la muerte fuese llevada a cabo sin dolor y si, de otra forma, el nuevo individuo consciente no existiría entonces la muerte del primero sería irrelevante y podría llevarse a cabo con cualquier fin (S. F. Sapontzis 1987, pp. 177-198).

$8 \mathrm{Al}$ margen de la propuesta singeriana, una referencia ineludible del análisis cualitativo del valor de las vidas la encontramos en J. S. Mill. Su idea es que hay placeres que difieren no ya desde el punto de vista de la cantidad sino desde el de la calidad. Estos hacen que el tipo de existencia humana sea cualitativamente más valiosa. Su propuesta en torno a cómo valorar vidas diferentes puede encontrarse en el capítulo 2 de su obra El utilitarismo. Véase J. S. Mill 1984, pp. 44-75.

9 Singer entiende la autoconciencia como aquello que implica el grado de racionalidad suficiente para poder tener una comprensión mínimamente racional del mundo, en línea con la propuesta de J. Locke. Véase J. Locke 1980, p. 492, cit. en P. Singer 2002, p. 101.

10 Del asunto de la personeidad se ha ocupado ampliamente Joel Feinberg. Para ahondar en su análisis véase J. Feinberg 1980. 
Singer ve claro que la aplicación de este argumento a los seres meramente conscientes está en línea con nuestras intuiciones al respecto. ${ }^{11}$ Los individuos autoconscientes, afirma, estarán sin embargo en un plano diferente. Son irremplazables porque quitarles la vida es privarles de aquello que han estado planeando conseguir, es privarles del resultado de sus esfuerzos.

Ello le lleva a plantear el modelo del viaje. ${ }^{12}$ Se trata de entender las vidas como viajes, situados en distintas etapas, en los que se ha invertido diferentes cantidades de esfuerzo para conseguir unos objetivos. El modelo es útil para hacerse cargo de algunas de nuestras intuiciones (P. Singer 1995, p. 161 y 1999, p. 57). Por ejemplo permite entender la decisión de no traer al mundo un niño como algo equivalente a impedir que se inicie un viaje. No habría que considerar demasiado alto el grado de daño implicado en este caso, y la razón es que al no haberse iniciado el viaje todavía no se ha tenido oportunidad de establecer objetivos y hacer planes al respecto. Una vez existimos, y en función de nuestras capacidades, podemos encaminar nuestro viaje hacia nuestras expectativas. Conforme avanzamos habremos invertido más esfuerzo en aumentar la probabilidad de alcanzar lo que queremos. Esto es una razón para admitir que el estadio donde se sitúa el viaje está en relación con el grado de daño que supondrá acabar con una vida. Cuanto más cerca estemos de conseguir aquello a lo que aspiramos peor hemos de considerar el hecho de que nuestro viaje acabe. Por ello habría que considerar los casos en los que la vida se interrumpe hacia el final de la misma como casos menos trágicos que si ello sucediera antes. La razón es que, en los estadios finales de la vida, los objetivos y planes hechos al comienzo del viaje, o bien ya se habrán conseguido o bien quedan escasas probabilidades de éxito al respecto.

La clave está en que para Singer los autoconscientes son los únicos que pueden concebir su propia existencia futura, ${ }^{13}$ y ello es lo que les permite plantear metas y objetivos a largo plazo. De forma que sólo de ellos puede decirse que han emprendido el viaje de su vida. La relevancia de la metáfora viene dada

11 Con el objetivo de justificar este argumento Singer comienza por distinguir dos posibles formas de llevar a cabo una reducción de la cantidad de placer en el mundo. Una consistiría en eliminar los placeres de los que llevan una vida placentera y la otra en eliminar a los individuos en sí que llevan vidas placenteras. Para profundizar en el análisis véase P. Singer 1995, pp. 126130, 148-163.

12 Este modelo descansa en la metáfora shakesperiana de la vida como viaje incierto. Véase P. Singer 1995, pp. 161-163.

13 Roger Crisp pensó en un primer momento que Singer estaba remarcando el importante papel que tendría en la evaluación la exclusiva capacidad de las personas humanas para otorgar un significado a sus vidas. Algo que el propio Singer se encargó de desmentir. Véase R. Crisp 1999, p. 86 y P. Singer 1999b, p. 288. 
por la importancia que Singer concede a la idea de vida biográfica. ${ }^{14}$ Este tipo de vida es una a la que sólo tendrán acceso los individuos racionales, autoconscientes y autónomos. Se trata de que puedan verse a sí mismos existiendo en el futuro. Sólo de los que sean capaces de ello podremos decir que se encuentran embarcados en su propio viaje de vida.

Pretendo señalar las graves dificultades que se le presentan a Singer a la hora poder justificar, desde el contexto normativo del utilitarismo de la preferencia, una estricta demarcación entre el tipo de tratamiento que merecen unos y otros individuos en torno a la muerte. Defiendo que, si quiere seguir partiendo de una teoría utilitarista, no podrá justificar una protección frente a la muerte en exclusiva para los autoconscientes, ni por tanto la existencia de diferencias cualitativas entre estos y los individuos conscientes. Por un lado el problema es que los intereses de ambos, sean más o menos complejos, parece que deberían ser igualmente tenidos en cuenta. Por otro no se entiende por qué las preferencias de autoconscientes no podrían ser reemplazadas por otras del mismo tipo pertenecientes a otros individuos autoconscientes. ${ }^{15}$

El autor tendría que explicar por qué, dentro del utilitarismo de la preferencia que caracteriza su propuesta, la satisfacción de los deseos del autoconsciente reemplazante no puede compensar la frustración de los deseos del autoconsciente reemplazado. La idea defendida por Singer acerca de la utilidad como satisfacción de preferencias y de la persecución de su maximización choca con la tajante distinción que quiere establecer en torno a distintos grados de conciencia. No parece fácil en un esquema como éste justificar una separación en función de las capacidades. Otra cosa es si se lograra mostrar por qué no puede darse la apuntada compensación entre preferencias. El autor insiste en que el modelo del viaje nos ayudará a percatarnos de la compatibilidad de los postulados utilitaristas con la negación de la reemplazabilidad para los autoconscientes. Pero lo que no está claro es cómo este modelo puede ayudar a evitar que la frustración de los deseos de unos pueda ser compensada (asumiendo un punto de vista global) por la satisfacción de los deseos de otros. Esto es, aunque el modelo del viaje resulta intuitivo, no queda claro que pueda funcionar como una buena razón para impedir que el argumento de la reemplazabilidad pueda

14 Un concepto que introduce James Rachels y que Singer retoma en un sentido más estricto. Consúltese J. Rachels 1990, pp. 199, 205 y P. Singer 1995, pp. 161-162. Puede verse un análisis al respecto en R. Fjellstrom 2003, p. 97.

15 La teoría, en los casos que prevé el argumento de la reemplazabilidad, debería demostrar indiferencia ante el hecho de que unos individuos sean sustituidos por otros. Será así aún cuando se haya adoptado una concepción del valor en la que cuente algo más que las sensaciones. Algo que dista mucho de cómo intuitivamente entendemos que hemos de valorar la vida humana. Véase F. Lara 2005, pp. 75-76. 
también aplicarse en el caso de los individuos autoconscientes. No se ve cómo un utilitarista podría justificar que hay algo malo en la desaparición indolora de un individuo, independientemente de su grado de conciencia, si se da la simultánea aparición de otro con una vida con el mismo grado de felicidad. Volviendo a la metáfora, habría que preguntar si acaso un viaje no podría ser potencialmente reemplazable por otro en el que haya los mismos elementos en juego. O por qué un viaje largo no podría ser reemplazado por varios viajes más pequeños (K. Smith 2002, pp. 55-71). El componente agregativo presente en la propuesta teórica de Singer apuntaría en dicho sentido.

Por otro lado el autor también reconoce la necesidad de hacerse cargo de un segundo objetivo en la discusión en torno al daño de la muerte. Es consciente de la necesidad de llevar a cabo justificadamente una toma de decisiones en la que haya que evaluar el valor de la vida de distintos individuos autoconscientes. Pero dentro del mismo esquema utilitarista igualmente será difícil poder mostrar que la preferencia por seguir viviendo de unos individuos autoconscientes pesa más que la de otros.

En este segundo contexto sustituye la idea de las diferencias cualitativas por la de que el valor de la vida de los distintos individuos autoconscientes debe ser visto como una función de la cantidad de intereses que cada uno puede contener dadas sus capacidades. Teniendo esto presente el autor apuesta por buscar alguna base neutral desde la que comprobarlo haciendo una comparación, y con tal fin nos propone un experimento mental (P. Singer 1995, pp. 131-135). Se trataría de que imagináramos que podemos convertirnos en un animal, un caballo por ejemplo. Cuando estamos en tal estado todo lo que podemos experimentar será lo mismo que experimentaría el animal en cuestión. Es decir, olvidamos completamente nuestra condición de humanos. Igualmente, cuando somos humanos todas nuestras experiencias serán únicamente las que experimentan los humanos. Nos pide además que imaginemos que tenemos también la facultad de adoptar un tercer estado en el que recordamos todas nuestras experiencias como humano y como animal. Si en esta tercera situación nos proponen elegir una de las dos existencias, sin duda, mantiene el autor, nos quedaríamos con la humana. Este experimento mental sería un mecanismo neutral mediante el que sería posible comparar de forma imparcial la existencia de cualquier animal con la existencia humana. Singer entiende que aunque este mecanismo pueda resultar problemático en algún sentido, no obstante nos permite comprobar que también dentro de la clase de individuos autoconscientes unas vidas son preferibles a otras.

Mediante este método se pretende justificar una ordenación del valor de las vidas de estos individuos sin caer en el especieísmo. Pero habrá que ver si este es un método satisfactorio. A la vista está la escasa funcionalidad del experimento mental propuesto. Aunque el ejemplo de los tres estados parece 
obedecer a la pretensión de jerarquizar las vidas de los autoconscientes sin tener que abandonar las coordenadas propias del utilitarismo estamos ante un experimento para el que los filósofos de la mente encuentran muchas dificultades. No parece fácil aceptar la posibilidad de que experimentemos en algún momento como un animal no humano. Además no se comprende bien cómo sería posible un tercer estado evaluativo neutro. ${ }^{16}$ Siendo realistas parece que, ni podemos experimentar plenamente la vida tal como la experimenta otra especie, ni podemos situarnos en ese contexto en que somos completamente neutrales en nuestra evaluación.

Al margen de la evaluación de la situación hipotética, lo importante es que Singer parece estar defendiendo la idea de que la vida de los más autoconscientes (como es el caso de los humanos normales) es más valiosa. Y no porque se den elementos cualitativamente superiores sino por la mayor cantidad de experiencias a las que se puede optar dadas las capacidades que caracterizan tal tipo de existencia. De manera que en este segundo paso de sus análisis, donde quiere establecer una jerarquía del grado de daño de la muerte para los diferentes individuos autoconscientes, parece estar pensando que la muerte de los más autoconscientes implica frustrar una mayor cantidad de preferencias ( $\mathrm{P}$. Singer 1995, pp. 136-145 y 1998, pp. 219-221). Ello es lo que hace que su vida sea más valiosa y por lo que hay considerar peor la muerte de los individuos que cuentan con un grado más alto de autoconsciencia. Sin embargo, de nuevo, dado el esquema utilitarista en que se encuentra no es baladí la pregunta acerca de por qué la preferencia por vivir de un perro pesaría menos que la de un humano. Como afirma Francisco Lara: «Tal vez la persona crea que resultará más perjudicada si no cumple sus objetivos porque considera que escribir un libro es más importante que dormitar, pero seguramente no lo será para el perro. Esto demuestra que el utilitarismo también es incapaz de evitar los conflictos entre intereses de seres de igual entidad moral y eso se debe en gran medida a su concepción bienestarista del bien (como satisfacción de deseos o preferencias), de la que sólo podría prescindir al precio de dejar de ser la misma teoría ética» (F. Lara 2006, p. 126).

He querido mostrar que el análisis de Singer resulta problemático en su pretensión de justificar una estricta demarcación para aquellos que han de estar moralmente protegidos frente a la muerte. No se lograba ver cómo, dentro de su propuesta ética, sería posible justificar la delimitación entre individuos

16 Roger Fjellstrom señala otros aspectos más concretos que cuestionan el buen diseño del experimento mental de los tres estados. En uno de los casos se pregunta el porqué de que el experimento sólo se centre en las versiones óptimas de las vidas de las diferentes especies. Ésta y otras cuestiones similares de corte metodológico se pueden encontrar en R. Fjellstrom 2003, p. 95 . 
conscientes y autoconscientes en este sentido. No queda probada la posibilidad de librar a las personas del ámbito del argumento de la reemplazabilidad. La metáfora del viaje, aunque intuitiva, no resulta útil en este sentido. Y en torno a la pretensión de jerarquizar el valor de las vidas, las mismas constricciones normativas dificultaban el objetivo. Al autor le resulta difícil poder defender la vida de los humanos normales como evaluativamente superior sin hacerlo desde lo que podría considerarse como una óptica humana en la que se está adoptando una perspectiva teórica que favorece «per se» tal tipo de existencia. ${ }^{17}$

\section{IV}

Además de los problemas de coherencia identificados en ambos contextos normativos, quiero insistir en la necesidad de no perder de vista el lugar común que comparten las dos propuestas presentadas con relación al análisis del daño de la muerte. Tanto Regan desde una teoría de los derechos, como Singer desde el utilitarismo de la prefencia, defienden una interpretación en la que lo que cuenta es la capacidad de valorar la propia vida si se quiere afirmar que la muerte indolora supone un daño para el individuo que muere. Lo que quiero mantener es que la interpretación descrita lleva a algunos resultados tan contraintuitivos que habría que repensar la noción de daño barajada en ella.

Obligatoriamente hay que pensar en el grupo de aquellos humanos que son conscientes pero no autoconscientes, porque en este esquema su muerte tendría que considerarse moralmente irrelevante y ello implicaría una desprotección moral al respecto. Los llamados casos humanos marginales ${ }^{18}$ (por ejemplo bebés, muchos discapacitados cognitivos graves o enfermos avanzados de Alzheimer) no tienen las capacidades que se asocian al grado de autoconciencia requerido. Sin embargo parece que no estamos dispuestos a asumir que la muerte indolora de esa clase de humanos es irrelevante en términos morales.

Podría esgrimirse que si nos situáramos en un marco normativo desde el que pudieran reconocerse razones indirectas entonces tendríamos una herramienta que nos permitiría ser más cautos. Podrían funcionar como razones de ese tipo las preferencias de los familiares al cuidado de los humanos marginales, que

17 Fjellstrom está interesado en mostrar que las conclusiones defendidas por Singer en torno al valor de las vidas no se sostienen. Mantiene que el cierto grado de favoritismo hacia los humanos que puede constatarse en la argumentación de Singer implicaría que finalmente no logra justificar su pretensión de no parcialidad, o bien que en algún momento concreto abandona tal pretensión. Véase R. Fjellstrom 2002, pp. 339-352 y 2003, pp. 91-106. También son muy interesantes las críticas a Singer que tanto B. Steinbock como R. J. Arneson elaboran en el mismo sentido. Consúltese B. Steinbock 1978 y R. J. Arneson 1999, p. 105.

18 Para algunos autores esta terminología es denigrante y apuestan por abandonarla. Véase por ejemplo H. Miller y W. Williams 1983; D. A. Dombrowski 1997; y S. D. Wilson 2005. 
estarían interesados en que las vidas de estos contaran con protección moral. O podría pensarse que hay que dar alguna relevancia al sentimiento tan fuerte de desagrado que nos produce la idea de tener que negar una protección moral frente al homicidio a esos humanos, que siendo conscientes no son tan racionales como los autoconscientes (B. Steinbock 1978). Sin embargo aún habría que preguntar si este es el tipo de protección que queremos para ellos. La contingencia de las razones indirectas junto con la fuerte intuición acerca de que no tendríamos que poder disponer de la vida de un discapacitado psíquico a nuestro antojo (por el individuo mismo), me llevan a pensar que algo falla en la elección del criterio descrito para determinar cuándo la muerte supone un daño.

A los problemas de coherencia asociados a las propuestas normativas analizadas se añade ahora la dificultad para justificar nuestras intuiciones prerreflexivas en torno a los casos de humanos meramente conscientes. Hay quien ha insistido en que el hecho de que este tipo de argumentación acerca del valor de la vida lleve a la anterior conclusión tendría que ser suficiente para poder afirmar que el procedimiento seguido resulta poco apropiado (A. Maclean 1993, pp. 17-36). Podríamos pensar que no se trata sólo de identificar los rasgos que confieren valor a la vida de un individuo y determinar qué individuos los poseen, por habitual que sea este método. Porque el problema de establecer previamente una característica y pasar a aplicarla a los casos particulares es que habitualmente ello supondrá muchos problemas a la hora de poder dar este segundo paso (J. Harris 1985).

Entiendo que resultará irrenunciable preguntar si la concepción de daño que hay debajo de las propuestas analizadas es la correcta para responder a la pregunta por cuándo la muerte supone un daño. Es fácil comprender las razones de Regan y Singer a la hora de querer justificar que la vida de los más autoconscientes es más valiosa, pero no puede pasarse por alto que en sus propuestas aparecen graves problemas difíciles de resolver e imposibles de pasar por alto. Puede que la clave esté en entender que se podría estar de acuerdo con tales razones sin que ello implique tener que comprometerse con la interpretación del daño presente en sus análisis. Defiendo que habría que perseguir una alternativa mejor, que evite los problemas de coherencia presentados, que se haga cargo de las intuiciones prerreflexivas que quieren justificar los autores analizados y que permita también reconocer que la muerte de los humanos meramente conscientes no es moralmente irrelevante. En este sentido mantengo que podría ser útil contar con la distinción entre daños intrínsecos y extrínsecos (T. Nagel 1970 y 2000). El objetivo sería justificar la adopción de una teoría amplia del daño desde la que fuera posible afirmar que algunas cosas pueden ser malas para nosotros aunque no se trate de cosas que experimentamos en sí mismas. Es decir, podría pasar a entenderse que algo podría ser malo porque supone un impedimento para que ocurran cosas intrínsecamente valiosas, y que concretamente ese sería el caso 
de la muerte. Adoptar este análisis privacionista del daño de la muerte llevaría a admitir que el hecho de continuar existiendo es moralmente relevante, no por nuestra preferencia al respecto sino porque ello es condición de posibilidad de experiencias valiosas para los individuos conscientes.

\section{REFERENCIAS BIBLIOGRÁFICAS:}

ARNESON, R. J. 1999: «What, if anything, renders all humans morally equal?», en D. Jamieson (Ed.): Singer and his critics. Oxford: Blackwell Publishers.

CAVALIERI, P. 2001: The animal question. Why nonhuman animals deserve human rights. Oxford: Oxford University Press.

CIGMAN, R. 1976: «Why death does not harm animals», en T. Regan y P. Singer (Eds.): Animal rights and human obligations. New Jersey: Prentice Hall.

_ 1981: «Death, misfortune and species inequality». Philosophy \& Public Affairs, 10.

CRISP, R. 1999: «Teachers in an age of transition: Peter Singer and J.S. Mill», en D. Jamieson (Ed.): Singer and his critics. Oxford: Blackwell Publishers.

DOMBROWSKI, D. A. 1997: Babies and beasts. Urbana: University of Illinois Press.

FEINBERG, J. 1980: «Abortion», en T. Regan (Ed.): Matters of life and death, New introductory essays in moral philosophy. New York: Random House.

FJELLSTROM, R. 2002: «Equality does not entail equality across species». Environmental Ethics, 24. 2003: «Is Singer's ethics speciesist?». Environmental Values, 12.

FREY, R. 1987: «Autonomy and the value of animal life». The Monist, 70.

HARRIS, J. 1985: The value of life. London and New York: Routledge.

HORTA, O. 2007: Un desafío para la bioética. La cuestión del especismo. Universidad Santiago de Compostela.

JAMIESON, D. 1990: «Rights, Justice and duties to provide assistance: a critique of Regan's theory of rights». Ethics, 100.

KANT, I. (1775-1781) 1988: Lecciones de ética. Barcelona: Crítica.

LARA, F. D. 2005: «Cuando dar la vida y quitarla es una cuestión de cálculo utilitario». TÉdos. Revista Iberoamericana de Estudios Utilitaristas, 14.

-2006: «La entidad moral de los animales y nuestras obligaciones con ellos». Signos Filosóficos, 8.

LOCKE, J. (1690) 1980: Ensayo sobre el entendimiento humano. Madrid: Editora Nacional.

MACLEAN, A. 1993: The elimination of morality. Reflections on utilitarianism and bioethics. London and New York: Routledge.

MILL, J. S. (1863) 1984: El utilitarismo. Madrid: Alianza Editorial.

MILLER, H. y WILLIAMS, W. (Eds.) 1983: Ethics and animals. Clifton, N. J.: Humana. 
NAGEL, T. 1970: «Death». Noûs, 4.

(1981) 2000: La muerte en cuestión. Ensayos sobre la vida humana. México: Fondo de Cultura Económica.

NUSSBAUM, M. (2006) 2007: Las fronteras de la justicia. Consideraciones sobre la exclusión. Barcelona: Paidós.

RACHELS, J. 1990: Created from animals. The moral implications of Darwinism. Oxford: Oxford University Press.

REGAN, T. 1983: The case for animal rights. Berkeley: University of California P. 1999: «Poniendo a las personas en su sitio». Teorema, 18.

ROSS, W. D. 1930: The right and the good. Oxford: Clarendon Press.

SAPONTZIS, S. F. 1987: Morals, reason and animals. Philadelphia: Temple University Press.

SINGER, P. (1975) 1999: Liberación animal. Madrid: Trotta.

— (1979) 1995: Ética práctica. Cambridge: Cambridge University Press.

— 1998: «Los animales y el valor de la vida», en T. Kwiatkowska y J. Issa (Comp.): Los caminos de la ética ambiental. México: Plaza y Valdés.

_ 1999a: «La ética más allá de los límites de la especie». Teorema, 18.

— 1999b: «A response», en D. Jamieson (Ed.): Singer and his critics. Oxford: Blackwell Publishers. (2000) 2002: Una vida ética. Escritos. Madrid: Taurus.

SMART, J. J. C. y WILLIAMS, B. 1973: Utilitarianism: for and against. Cambridge: Cambridge University Press.

SMITH, K. 2002: «Animal genetic manipulation: a utilitarianism response». Bioethics, 16 (1).

STEINBOCK, B. 1978: «Speciesism and the idea of equality». Philosophy, 53 (204).

WILSON, S. D. 2005: «The species-norm account of moral status». Between the Species, 5. 
OLGACAMPOS SERENA es doctora en Filosofía por la Universidad de Granada. Actualmente se encuentra en Oxford Uehiro Centre for Practical Ethics. (University of Oxford, UK) con un contrato posdoctoral CEI (Campus de Excelencia Internacional).

\section{Publicaciones recientes:}

«La mejora del carácter moral en la evaluación de la técnicas de mejora biológica». Dilemata: Revista Internacional de Éticas Aplicadas, 3 (2010). (ISSN 1989-7022)

«¿La muerte daña al que muere? Experiencia y privación», en A. Bermejo Salar, et ál.(Eds.): Umbrales Filosóficos. Posicionamientos y perspectivas del pensamiento contemporáneo. Murcia: Edit.um, 2011. (ISBN 978-84-8371-302-0)

Línea de investigación:

Bioética, Derechos Morales de los Animales, Tanatoética.

Dirección postal institucional:

Departamento de Filosofía I

Facultad de Psicología

Universidad de Granada

Campus Cartuja

18071 Granada (Spain)

Dirección electrónica: olgacampos@ugr.es 
